appropriately. I suspect that high $R^{2}$ values would be obtained if this category were used as the predictor variable, which would lead to rejection of the assumed independence between depression and KSP. Furthermore, the effect of SSRIs on depression would then be a more plausible explanation of the changes in personality traits during antidepressant treatment.

The 'normalising' effect of treatment on KPS requires that regression towards the mean is thoroughly evaluated. The authors dismiss it by pointing out that previous findings are in line with their results. This is an invalid argument. Two methods that can be used to detect this phenomenon come to mind. One consists of evaluating the difference between the two measures of each subject against the arithmetic mean of the measures of each subject, rather than the difference between the two measures against the initial measure as considered by Ekselius \& von Knorring. This technique is neither infallible nor a substitute to the second method, that is the use of a control group. Ekselius \& $x$ von Knorring do not have a control group, which means to me that the study's design is inappropriate for its secondary aim, the measurement of a treatment effect, and its conclusions lack sufficient logical support to be generalised.

Evelina, L \& von Knoming, L. (1989) Changes in personality traits during treatment with sertraline or citalopram. British journol of Psychiotry, 174, 444-448.

D. Marchevaky Department of Psychiatry, Campbell Centre, Hospitals Campus, Standing Way. Eaglestone, Milton Keynes MK6 5NG

Authors'reply: Dr Marchevsky finds it difficult to accept the assumed independence between depression and the majority of the self-rated Karolinska Scales of Personality (KSP). However, the empirical data are in favour of such an assumption. In an earlier study (Perris et al, 1979), in-patients suffering from a moderately severe or severe depressive syndrome completed the KSP on two separate occasions. The first investigation was carried out shortly after the patients' admission to hospital when they were depressed, and the second a few months later when they were at home having recovered from the depressive illness. Most of the patients were at that time treated with tricyclic antidepressants or electroconvulsive therapy. The results of that study verified that the inventory measures traits which are fairly independent of the state of the subject. However, significant differences were seen between the two assessments for psychic anxiety, somatic anxiety and social desirability, which is why changes in these scales should be interpreted with caution.

Second, Dr Marchevsky gives an example in which patients with large MADRS changes have small KSP changes while patients with small MADRS changes have large XSP changes. In our opinion, such an example would strongly go against a relationship between the changes in depressive symptomatology and the changes in personality traits.

Finally, Dr Marchevsky raises the question concerning regression towards the mean. He discusses mathematical strategies to handle the problem but concludes that this technique is neither infallible nor a substitute to the second method - the use of a control group. We agree.

He concludes that there is not enough support to generalise the results. We agree, as is clear from the tentative wording of our Clinical Implications (Ekselius \& von Knorring, 1999, p. 448).

Thus, we still believe that there is support for our conclusions, although a limitation that we pointed out (Ekselius \& von Knorring, 1999, p. 448) must be borne in mind: "Although the personality inventory used has been demonstrated to be fairly independent of the clinical state of the investigated subjects, the improvement in depressive symptomatology may have influenced the results".

Eccellus, L. \& von Knorring, L. (19\%) Changes in personality traits during treatment with sertraline or citalopram. British joumol of Psychiatry, 174. 444-448.

Perris, C., Elsemann, M., Eriksson, U., et ol (I979) Variations in self-assessment of personality characteristics in depressed patients, with special reference to aspects of aggression. Psychiatrica Clinico, 12, 209-215

L. Ekselius, L. von Knorring Department of Neuroscience, Psychiatry, University Hospital, SE-75I 85 Uppsala, Sweden

\section{More on amiodarone-induced depression}

Sir: Ambrose \& Salib (1999) assume that the depression induced in their patient by treatment with amiodarone, may not have been due to effects on thyroid functioning. Amiodarone, however, produces a variety of effects on thyroid hormone physiology.

Amiodarone reduces the conversion of thyroxine $\left(\mathrm{T}_{4}\right)$ to triiodothyronine $\left(\mathrm{T}_{3}\right)$, results in a modest decrease in $T_{3}$ serum levels, and a rise in $T_{4}$ and reverse $T_{3}$ levels. Thyroid-stimulating hormone (TSH) levels rise transiently in the first three months but thereafter return to normal. These effects are similar to the results obtained by Ambrose \& Salib of raised $\mathrm{T}_{4}$ and normal TSH levels after eight months of amiodarone treatment.

The majority of patients treated with amiodarone remain clinically euthyroid, albeit with altered thyroid indices, as in Ambrose \& Salib's patient. However, occasional patients may develop overt or subclinical hypothyroidism or hyperthyroidism. In subclinical hypothyroidism clinical symptoms are generally absent, however some studies have reported somatic and psychiatric symptoms such as depressed mood, lethargy, obssessionality and impaired cognitive function. Lithium, another drug which inhibits thyroid hormone release, has been reported to cause thyroid dysfunction even when thyroid function test results remain within normal limits.

It has been suggested that hypothyroidism is a graded phenomenon with varying degrees of clinical severity and biochemical abnormality. It may well be that amiodaronetreated patients, although outside the range for overt hypothyroidism, still have subtle abnormalities in thyroid function. Observations in animal models suggest that amiodarone can antagonise thyroid hormone action at the cellular level by blocking hormone entry and receptor binding. The mildly altered biochemical indices seen in euthyroid patients on amiodarone may represent compensatory effects. Thus, the increased $\mathrm{T}_{4}$ level may serve to overcome the blockade of $T_{3}$ production, thereby maintaining normal $T_{3}$ levels, which, in turn, may help to restore TSH levels to normal.

Ambrose \& Salib's report is important as it is perhaps only the second report of psychiatric symptoms induced by amiodarone. However, they were quick to dismiss the role which may have been played by thyroid function.

Ambrose, A. \& Salib, E. (1999) Amiodarone-induced depression. British journal of Pyychiatry, 174, 366-367.

Ftre, H. L. \& Fi:ce, f. (1990) The effects of amiodarone on thyroid hormone function: a review of the physiology and clinical manifestations. fournal of Clinicol Phormocologx 30, 588-595.

Kleiner, J., Attahuler, L., Hendrick, V., ex of (19s9) Lithium induced subclinical hypothyroidism: review of the literature and guidelines for treatment. joumd of Clinical Psychiotry, 60, 249-255. 
Nicholeon, J. (ISM) Amiodarone and psychiatric symptoms (letter). British journal of Psychiotry. 175 . $191-192$.

Repnolds, J. E. F. (19s) Mortindale: The Extro Pharmocopoeia. London: Royal Pharmaceutical Society.

A.T. Odelola Rawnsley Building, Manchester Royal Infirmary, Oxford Road, Manchester MI3 9WL

\section{Venlafuxine and paroxetine in treatment-resistant depression}

Sir: Poirier \& Boyer (1999) suggest their comparative trial showed some evidence of superiority of venlafaxine over paroxetine in treatment-resistant depression. Examining the previous antidepressants used throws considerable doubt on this conclusion.

Sixty-six per cent of the venlafaxine group and $65 \%$ of the paroxetine group had previously been treated with a selective serotonin reuptake inhibitor (SSRI). As venlafaxine is the only serotonin-noradrenaline reuptake inhibitor (SNRI) available, none of the patients will have previously taken an SNRI. Most of the patients in the study have already found one SSRI ineffective: we should not be surprised that paroxetine tended to be less helpful.

Although numbers would be small it would be interesting to examine response rates for the patients who had not been treated with an SSRI previously. I suspect a difference may not be apparent in this group.

Despite reservations, I note that the study does throw some light on a common clinical problem. Psychiatrists generally look to drugs of a different class when one medication has been unhelpful. This study supports this practice, at least in the use of venlafaxine rather than another SSRI when treatment with a first SSRI has failed.

Polrier, M. F. \& Eoyer, P. (ISs) Venlafaxine and parocetine in treatment-resistant depression. Doubleblind, randomised comparison. British journol of Psychiatry 175, 12-16.

J. Gregson Alfred Child and Adolescent Mental Health Service, 594 St Kilda Road. Melbourne, Victoria 3004, Australia

Authors' reply: In response to Dr Gregson, while approximately two-thirds of patients in the venlafaxine and paroxetine groups had received an SSRI previously, $69 \%$ of the venlafaxine group and $73 \%$ of the paroxetine group had also received a tricyclic antidepressant (TCA) previously. No differences in response were noted between treatment groups among patients previously treated with a TCA. Although not strictly considered as SNRIs, the TCAs exhibit a range of activity on serotonin, noradrenaline, or combined serotoninnoradrenaline reuptake.

We agree with Dr Gregson that the results of our study support the use of an antidepressant of a different pharmacological class in patients who have failed to respond to an SSRI.

M.-F. Poirler SHU-Centre Hospitalier SainteAnne, I rue Cabanis, 75674 Paris, France

P. Boyer Hôpital Pitié-Salpêtrière, Paris, France

\section{Urinary detection of olanzapine - an aid to compliance}

Sir: With increasing use of oral atypical antipsychotic drugs and the current absence of injectable preparations, it is to be expected that the use of depot medication will decline (Liberman, 1988). With this comes the increased importance of ensuring patient compliance with medication.

Previous analysis of serum olanzapine levels has primarily been concerned with the pharmacokinetics and clinical response to particular levels (Perry et al, 1997; Prieto et al, 1997; Berna et al, 1998), and these results are, as yet, not useful for everyday clinical practice.

We report that in a carefully selected group of patients, monitoring of the presence or absence in urine of a particular antipsychotic may help to confirm compliance with prescribed medication. This will provide greater confidence (in professionals, the patient and the community) that people are being treated for their mental disorder appropriately. It is important to state that at this stage we are looking at a qualitative test for presence or absence of the drug, not a quantitative test, which may have more relevance to efficacy and effectiveness of treatment. The use of urinary testing, rather than blood testing, should generally be more acceptable to patients. It should also help to ensure that there is no confusion between periodic urinary testing for the presence of olanzapine and the routine blood monitoring that is required for potentially dangerous drugs such as clozapine.

The method currently used to screen for olanzapine involves gas chromatography/ mass spectrometry, and concentrations can be detected down to at least $100 \mu \mathrm{g} / \mathrm{l}$ using this method (I. Marsh, personal communication, 1999). Urine ( $5 \mathrm{ml}$ ) is extracted using a Toxilab extraction tube, Toxi-tube A (Microgen Products, Lake Forest, CA). The organic extract is evaporated to dryness under nitrogen and reconstituted in $100 \mu \mathrm{l}$ butyl acetate. The extract is then analysed by gas chromatography/mass spectrometry using a Hewlett-Packard HP6890/5973 system. Extract $(1 \mu \mathrm{l})$ is injected onto a HP-SMS capillary column $(30 \mathrm{~m} \times 0.25 \mathrm{~mm} \times 0.25 \mu \mathrm{m})$ and analysed in scan mode using a temperature program starting at $85^{\circ} \mathrm{C}$ and ramping to $280^{\circ} \mathrm{C}$ at $10^{\circ} \mathrm{C}$ per minute with a final temperature hold of nine minutes. Olanzapine is then identified by retention time and mass spectral data.

The normal elimination half-life of 32.4 hours for olanzapine should allow variable or inconsistent compliance to be detected within a reasonable period of time. The absence of clinically active metabolites further strengthens the appropriateness of the test. A mass balance study showed that approximately $57 \%$ of radiolabelled olanzapine appeared in the urine, principally as metabolites (data provided by manufacturer).

We believe we may be the first to use clinically a form of testing of urine for the presence of olanzapine. The mode of monitoring has been to discuss the situation with patients when they are in remission, to ask whether they agree to submit to random testing at various points in their community care. Obviously, patient consent can be withdrawn at any time, which may suggest that compliance is poor. If the result is positive, we have discovered that it has strengthened the therapeutic alliance between patient and doctor. We believe this is particularly valuable in cases where patients have previously shown a high degree of dangerousness when in relapse. The limitation is, of course, that quantification is not undertaken, which would mean that even very low urine levels may be detected as positive. However, as an initial first step to aiding compliance, or perhaps what is more correctly called concordance with antipsychotic medication, we believe that this form of therapeutic drug monitoring (Olesen \& Linnet, 1998) could present a major step 